\title{
Barrier Information Coverage with Wireless Sensors
}

\author{
Guanqun Yang and Daji Qiao \\ Iowa State University, Ames, IA 50011 \\ \{gqyang,daji\}@iastate.edu
}

\begin{abstract}
Sensor networks have been deployed for many barrier coverage applications such as intrusion detection and border surveillance. In these applications, it is critical to operate a sensor network in an energy-efficient manner so the barrier can be covered with as few active sensors as possible. In this paper, we study barrier information coverage which exploits collaborations and information fusion between neighboring sensors to reduce the number of active sensors needed to cover a barrier and hence to prolong the network lifetime. Moreover, we propose a practical solution to identify the barrier information coverage set which can information-cover the barrier with a small number of active sensors. The effectiveness of the proposed solution is demonstrated by numerical and simulation results.
\end{abstract}

\section{INTRODUCTION}

Applications such as intrusion detection and border surveillance [1] constitute a compelling application category for wireless sensor networks. The main goal of these applications is to detect targets as they cross a barrier, which is usually a long belt region. Sensors are deployed in the barrier to prevent targets from crossing the barrier undetected. This is often referred to as the barrier coverage problem [2], [3].

In this paper, based on the probabilistic sensing model, we study the barrier coverage problem under the assumption that neighboring sensors may collaborate with each other to form a virtual sensor which makes the detection decision based on combined sensed readings. This is also known as the barrier information coverage problem. Specifically, a point $t$ is said to be information-covered if, when a target is present at point $t$, it can be detected by at least one sensor with a probability larger than or equal to a pre-determined value $\left(P_{\mathrm{D}}^{\mathrm{min}}\right)$, while the system's false detection probability is smaller than or equal to a pre-determined value $\left(P_{\mathrm{FD}}^{\max }\right)$. We assume that the sensor locations are unknown to the target which follows a perpendicular crossing path to cross the barrier so as to minimize the probability of being detected. A barrier is said to be information-covered if, for any perpendicular crossing path, there exists at least one point along the path that is information-covered. We study how to operate a sensor network to information-cover a barrier efficiently.

The goal is to information-cover a barrier with as few active sensors as possible. This is different from the barrier coverage problem under the conventional $0 / 1$ disc sensing model because the size and shape of the coverage region of a virtual sensor vary with the decision threshold and the positions of collaborating sensors. Note that the size and shape of sensors' coverage regions affect the number of sensors needed to provide the coverage to the barrier, which in turn determines the decision threshold. Such mutual correlation makes this problem non-trivial and challenging.

To address this problem, we (i) map the barrier coverage problem to the line coverage problem by projecting sensors'

The research reported in this paper was supported in part by the Information Infrastructure Institute (iCube) of Iowa State University and the National Science Foundation under Grants CNS 0716744 and CNS 0831874. coverage regions on the destination side of the barrier, (ii) derive an effective lower bound to approximate the projection length of a virtual sensor's coverage region, and (iii) based on the derived lower bound, propose a practical solution to find a set of active sensors to information-cover the barrier. In-depth simulation study demonstrates the effectiveness of informationcoverage and sensor collaboration in reducing the number of active sensors needed to cover a barrier and the effectiveness of our proposed solution in finding a proper barrier information coverage set.

The rest of this paper is organized as follows. We discuss the related work in Section II. Then, we give the system models and the problem statement in Section III. Section IV studies the coverage region of a virtual sensor and its effect on the coverage performance and derives a lower bound for its projection length. Section V describes our proposed solution to find a BICS. Section VI presents the performance evaluation results and Section VII concludes the paper.

\section{RELATED WORK}

The area coverage problem based on the $0 / 1$ disc sensing model has been studied in the past [4]-[7]. In such a model, an object inside or outside a sensor's sensing range is detected by the sensor with probability one or zero. Recently, the barrier coverage problem [2], [3] has attracted great attention. Theoretical foundations for weak and strong barrier coverage in a randomly deployed sensor network are studied in [3], [8]. For a finite barrier, both [9] and [10] provide analytical methods to estimate the sensor network density for achieving barrier coverage and connectivity or measuring the quality of barrier coverage. Centralized and distributed algorithms for providing barrier coverage are proposed and evaluated in [11] and [12]-[14], respectively. All of the above works assume the 0/1 disc sensing model. Despite its simplicity of analysis, many researchers start to consider alternative sensing models in order to better study and characterize the sensor measurements which are usually affected by noise and vary with the distance between the sensor and the object. The works in [2], [15]-[17] assume the exposure model or the probabilistic sensing model to study the coverage and detection problem. Different from our study, these works do not consider sensors' collaborations and information fusion for coverage.

Collaboration among sensors [18] has been considered in many sensor network applications such as tracking and object detection. Some fundamental problems on information fusion for distributed detection are studied in [19]-[21] where the local data or decisions of individual sensors are gathered by a fusion center to make the final decision. Recently, in [22], the authors introduce the concept of virtual sensor resulting from neighboring sensors' collaboration based on value fusion, which may improve the coverage performance. In addition, [22] proposes a heuristic algorithm to select a sensor cover set from deployed sensors to information-cover the area. However, this algorithm does not adjust sensors' decision thresholds. 
Therefore, sensors' maximum coverage regions may not be fully exploited. Moreover, application of information coverage and virtual sensors to improve the barrier coverage has not been studied elsewhere.

\section{Models And Problem Statement}

\section{A. System and Source Models}

We consider a network of $N$ wireless sensors deployed to monitor a barrier which is a long belt region with two parallel sides: entrance side and destination side. Let $\mathcal{B}$ denote the barrier. We assume that sensors know their locations in $\mathcal{B}$ and the sensor network is always connected. In this paper, we study the targets that emit or cause physical signals such as sound, electromagnetic waves or seismic waves on the ground. The strength of the signal emitted or caused by the target decays according to power law, meaning that if the target is at point $t$, the signal strength measured by sensor $s_{i}$ is: [21], [22]

$$
\omega_{i}(t)= \begin{cases}\Omega, & d_{i}<d_{0}, \\ \frac{\Omega}{\left(d\left(s_{i}, t\right) / d_{0}\right)^{\alpha}}, & d_{i} \geqslant d_{0},\end{cases}
$$

where $\Omega$ is the signal amplitude at the target, $d_{0}$ is a small constant, and $\alpha$ is a known decay exponent. Here, we use $d(\cdot, \cdot)$ to denote the distance between two points. While the analysis and algorithm in this paper may be applied to any decay exponent, we are particularly interested in $\alpha=1$ or 2 . This is because, in typical barrier coverage applications such as border surveillance, human activity generates vibrations that propagate away from the source as seismic waves, whose energy is distributed in both Rayleigh waves (70\%) and body waves $(30 \%)$ [23], [24]; the signal strength decay exponents for these two waves are 1 and 2, respectively.

At each sensing cycle, every active sensor $s_{i}$ collects its sensed reading of $\mathbf{x}_{i} \cdot{ }^{1}$ Depending on the hypothesis of whether a target is present in the barrier $\left(\mathcal{H}_{1}\right)$ or not $\left(\mathcal{H}_{0}\right)$, and the position of the target $(t)$, the sensed reading is:

$$
\left\{\begin{array}{l}
\mathcal{H}_{0}: \mathbf{x}_{i}=n \\
\mathcal{H}_{1}: \mathbf{x}_{i}=\omega_{i}(t)+n,
\end{array}\right.
$$

where $\omega_{i}(t)$ is the received signal strength given by (1) and $n$ is the background noise. In this paper, we use $F_{N}(n)$ to denote the cumulative distribution function of noise, and assume that it is identical and independent for all sensors.

\section{B. Detection Model}

A sensor can make a detection decision solely based or its own sensed reading, or collaborate with nearby sensors tc make a decision based on combined sensed readings. There are two types of fusion algorithms for collaborative target detection [21] in wireless sensor networks: value fusion and decision fusion. In this paper, we investigate and analyze the value fusion-based collaborative target detection and define a virtual sensor as follows. We assume the "SUM" rule for a virtual sensor to combine sensed readings.

Definition 1 (Virtual Sensor): A virtual sensor $v_{j}$ is an entity that incorporates the sensed readings of a designated group of collaborating sensors $\left(s_{j_{1}}, \cdots, s_{j_{k}}\right)$ and makes a decision based on the combined sensed readings, where $k$ is the

\footnotetext{
${ }^{1}$ We assume that a detection decision is made based on snapshot readings. How to improve target detection and barrier coverage by considering temporal correlation among sensed readings will be studied in the future work.
}

collaboration degree of the virtual sensor. A target detection is claimed if the sum of sensed readings is larger than or equal to a pre-determined decision threshold, $T_{k}$ :

$$
\left\{\begin{array}{l}
\mathcal{H}_{0}, \text { if } \sum_{m=1}^{k} \mathbf{x}_{j_{m}}<T_{k} ; \\
\mathcal{H}_{1}, \text { if } \sum_{m=1}^{k} \mathbf{x}_{j_{m}} \geqslant T_{k} .
\end{array}\right.
$$

Note that when $k=1$, a virtual sensor is equivalent to a noncollaborating physical sensor (or noColl sensor for short).

A previous study [22] has shown that the performance improvement by raising the collaboration degree from two to three or higher is limited while the implementation complexity increases significantly. Therefore, we focus on the situation when the collaboration degree of a virtual sensor is at most two. In other words, we study a sensor network consisting of either virtual sensors with only two associated physical sensors or noColl sensors that do not collaborate with others.

DeFinition 2 (Sensing Intensity): The sensing intensity of a virtual sensor $v_{j}$ at point $t$ is $I_{j}\left(d\left(s_{j_{1}}, t\right), \cdots, d\left(s_{j_{k}}, t\right)\right)=$ $\sum_{m=1}^{k} \omega_{j_{m}}(t)$, where $\omega_{j_{m}}(t)$ is the signal strength measured by sensor $s_{j_{m}}$ - the $m$-th physical sensor associated with the virtual sensor $v_{j}$. For a noColl sensor $s_{i}$ that does not collaborate with other sensors, its sensing intensity at point $t$ is simply $I_{i}\left(d\left(s_{i}, t\right)\right)=\omega_{i}(t)$.

In this paper, in contrast to the $0 / 1$ disc sensing model, we consider the probabilistic sensing model where (i) the sensed readings are affected by noise, as shown in Eq. (2); and (ii) based on a pre-determined decision threshold, a target is detected by a virtual or noColl sensor with certain probability, which varies with the sensing intensity at the target's position. Fig. 1 illustrates the probability of detection $\left(P_{d}\right)$ and false detection $\left(P_{f}\right)$ by a sensor. We can see that $P_{d}$ increases with the sensing intensity $I$. For a virtual sensor with the collaboration degree of two, $\mathbf{x}$ is the sum of sensed readings by its two associated physical sensors, $T=T_{2}$ is the decision threshold, and $P_{f}=1-F_{2 N}\left(T_{2}\right)$. Note that $F_{2 N}(\cdot)$ is the cumulative distribution function of the sum of two independent noises. For a noColl sensor, $\mathbf{x}$ is its own sensed reading, $T=T_{1}$ is the decision threshold, and $P_{f}=1-F_{N}\left(T_{1}\right)$.

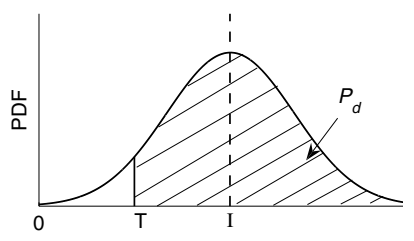

(a)

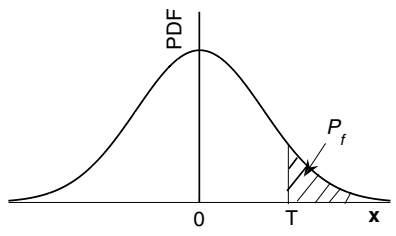

(b)
Fig. 1. (a) Probability of detection: $P_{d}=P\left(\mathbf{x} \geqslant T \mid \mathcal{H}_{1}\right)$ and (b) probability of false detection: $P_{f}=P\left(\mathbf{x} \geqslant T \mid \mathcal{H}_{0}\right)$.

Once a sensor makes a local detection decision, it sends the decision (a Boolean value) to the sink. Since the system has no information about where the target may appear, we adopt the "OR" rule to make the decision fusion, i.e., we claim that the target is present in the barrier if any virtual or noColl sensor reports a detection. Therefore, the system's false detection probability is

$$
P_{F D}=1-\prod_{i=1}^{M}\left(1-P_{f_{i}}\right),
$$


where $P_{f_{i}}$ is $1-F_{2 N}\left(T_{2}\right)$ for virtual sensors and $1-F_{N}\left(T_{1}\right)$ for noColl sensors, and $M$ is the total number of virtual or noColl sensors in $\mathcal{B}$.

\section{Coverage Region and Barrier Information Coverage}

DeFinition 3 (Point Information Coverage): Given the value fusion-based collaborative target detection described in the previous section, we say that a point $t$ is informationcovered if, when a target is present at point $t$, it can be detected by at least one sensor with a probability larger than or equal to a pre-determined value $\left(P_{D}^{\mathrm{min}}\right)$, while the system's false detection probability is smaller than or equal to a pre-determined value $\left(P_{F D}^{\max }\right)$.

From this definition, we know that a point $t$ is informationcovered by a noColl sensor $s_{i}$ if the sensing intensity of $s_{i}$ at point $t$ is larger than or equal to a certain threshold, denoted as $I_{\text {threshold_1 }}$ :

$$
\begin{gathered}
P_{d}=1-F_{N}\left(T_{1}-I_{i}\left(d\left(s_{i}, t\right)\right)\right) \geqslant P_{\mathrm{D}}^{\min } \\
\Longrightarrow I_{i}\left(d\left(s_{i}, t\right)\right) \geqslant T_{1}-F_{N}^{-1}\left(1-P_{\mathrm{D}}^{\min }\right) \triangleq I_{\text {threshold_1 }} .
\end{gathered}
$$

Accordingly, we can determine the coverage region for $s_{i}$ :

$$
\begin{aligned}
\mathcal{A}_{i} & \equiv\left\{t \mid I_{i}\left(d\left(s_{i}, t\right)\right) \geqslant I_{\text {threshold_1 }}\right\}=\left\{t \mid \omega_{i}(t) \geqslant I_{\text {threshold_1 }}\right\} \\
& =\left\{t \mid d\left(s_{i}, t\right) \leqslant d_{0} \sqrt[\alpha]{\left.\frac{\Omega}{I_{\text {threshold_1 }}}\right\} .}\right.
\end{aligned}
$$

Similarly, we can determine the coverage region for a virtual sensor $v_{j}$ with collaboration degree of two as follows. A point $t$ is information-covered by $v_{j}$ if the sensing intensity of $v_{j}$ at point $t$ is larger than or equal to a certain threshold, denoted as $I_{\text {threshold_2 }}$ :

$$
\begin{gathered}
P_{d}=1-F_{2 N}\left(T_{2}-I_{j}\left(d\left(s_{j_{1}}, t\right), d\left(s_{j_{2}}, t\right)\right)\right) \geqslant P_{\mathrm{D}}^{\min } \\
\left.\Longrightarrow I_{j}\left(d\left(s_{j_{1}}, t\right), d\left(s_{j_{2}}, t\right)\right)\right) \geqslant T_{2}-F_{2 N}^{-1}\left(1-P_{\mathrm{D}}^{\min }\right) \triangleq I_{\text {threshold_2. }} .
\end{gathered}
$$

Then, the coverage region for $v_{j}$ is

$$
\begin{aligned}
\mathcal{A}_{j} & \left.\equiv\left\{t \mid I_{j}\left(d\left(s_{j_{1}}, t\right), d\left(s_{j_{2}}, t\right)\right)\right) \geqslant I_{\text {threshold_2 }}\right\} \\
& =\left\{t \mid \sum_{m=1}^{2} \omega_{j_{m}}(t) \geqslant I_{\text {threshold_2 }}\right\} \\
& =\left\{t \mid \sum_{m=1}^{2} \frac{\Omega}{\left(d_{j_{m}} / d_{0}\right)^{\alpha}} \geqslant I_{\text {threshold_2 }}\right\} .
\end{aligned}
$$

It is interesting to see that the size and shape of the coverage region of a virtual sensor vary with the positions of the collaborating sensors, even when the source and noise models are fixed, unlike the coverage region of a noColl sensor, which is a disc with a fixed radius.

Definition 4 (Crossing Path): A path in $\mathcal{B}$ is said to be a crossing path if it crosses the complete width of $\mathcal{B}$, i.e., starts from any point on the entrance side of $\mathcal{B}$ and ends at any point on the destination side of $\mathcal{B}$. A crossing path is perpendicular if its length equals the barrier width.

DeFinition 5 (Information Coverage of a Path): A path is said to be information-covered if there exists at least one point on the path that is information-covered.

In this paper, we assume that sensor locations are unknown to the target. Previous studies [2], [3] have shown that, in order to minimize the probability of being detected by the sensor network in this situation, the target should follow a perpendicular crossing path to cross the barrier. We study how to operate a sensor network to prevent such targets from crossing the barrier without being detected. This is often referred to as the weak barrier coverage problem. By contrast, strong barrier coverage refers to the problem of deploying a sensor network to protect the barrier against targets that may follow any possible crossing path. Strong barrier coverage is a more challenging problem and will be studied in the future.

DeFinition 6 (Barrier Information Coverage): A barrier is said to be barrier-information-covered if all perpendicular crossing paths are information-covered.

DeFINITIon 7 (Barrier Information Coverage Set (BICS)): A BICS is a set of active sensors that can information-cover the barrier.

\section{Problem Statement}

Given a network of wireless sensors deployed in a barrier, and given the target probability of detection by a virtual or noColl sensor $\left(P_{\mathrm{D}}^{\mathrm{min}}\right)$ and the system's target false detection probability $\left(P_{\mathrm{FD}}^{\max }\right)$, our goal is to develop a scheme to find a BICS that consists of as few active sensors as possible, so as to prolong the sensor network lifetime.

\section{THEORETICAL ANALYSis}

To achieve our design goal, we first study the coverage region of a virtual sensor and its effect on the barrier coverage.

\section{A. Mapping Barrier Coverage to Line Coverage}

DeFinition 8 (Barrier Projection): The barrier projection of a set of points in the barrier is the set of the projections of these points on the destination side of the barrier.

Given Definition 6 on barrier information coverage and Definition 8 on barrier projection, it is straightforward to prove that a barrier is information-covered if and only if the barrier projections of the coverage regions of active sensors can cover the entire destination side of the barrier, as illustrated in Fig. 2. Proof of this statement is omitted due to space limitation (see [25] for details).

\section{B. Coverage Region of a Virtual Sensor}

Fig. 3 plots the coverage regions of virtual sensors with their associated physical sensors at different positions. In the figure, the $\mathrm{X}$-axis is in parallel to the destination side of the barrier. Let $L$ and $\theta$ denote the distance between two sensors and the angle between the line segment connecting two sensors and the destination side of the barrier, respectively. We have a couple of observations. Firstly, the size and shape of the coverage region of a virtual sensor vary with the positions of its two collaborating sensors, which can be characterized by $L$ and $\theta$. This conforms to our earlier discussion in Section III-C. Secondly, a virtual sensor consisting of two collaborating physical sensors may not always improve the barrier coverage performance than these two noColl sensors operating independently without collaborating with each other. For example, while the virtual sensor in Fig. 3(a) improves the barrier coverage performance than the non-collaborative case, the virtual sensor in Fig. 3(b) indeed degrades the barrier coverage performance, while the performance gain by using virtual sensors in Fig. 3(c) and (d) is minimal. So it is important 
to have a good understanding about the effect of $L$ and $\theta$ on the coverage region of a virtual sensor.

In general, it is difficult to express the coverage region of a virtual sensor (denoted as $\mathcal{A}$ ) and consequently the length of the barrier projection of the coverage region (denoted as $\left.\left|\mathcal{P}_{\mathcal{A}}\right|\right)$ as closed-form functions of $\theta, L$, and $\alpha$. Instead, we use some simple geometry graphs to construct $\mathcal{A}_{\text {sub }}$, a subset of $\mathcal{A}$, and use the length of the barrier projection of $\mathcal{A}_{\text {sub }}$ (denoted as $\left|\mathcal{P}_{\mathcal{A}_{\text {sub }}}\right|$ ) to approximate $\left|\mathcal{P}_{\mathcal{A}}\right|$. Without loss of generality, we first study $\mathcal{A}$ and $\mathcal{A}_{\text {sub }}$ by fixing $\theta$ to 0 in Section IV-C, because the size and shape of $\mathcal{A}$ do not vary with $\theta$. Then in Section IV-D, we study $\left|\mathcal{P}_{\mathcal{A}_{\text {sub }}}\right|$ for different $\theta$ values. Finally in Section IV-E, we use numerical study to evaluate the analytical results and the tightness of $\left|\mathcal{P}_{\mathcal{A}_{\text {sub }}}\right|$ as a lower bound to $\left|\mathcal{P}_{\mathcal{A}}\right|$.

\section{Finding $\mathcal{A}_{\text {sub }}$}

We study $\mathcal{A}$ and $\mathcal{A}_{\text {sub }}$ of a virtual sensor with the collaboration degree of two. In [25], we show that the coverage region of such a virtual sensor can be either connected or disconnected (with two disconnected symmetric components) depending on the distance between its two collaborating physical sensors. In other words, there exists a critical distance, $L_{\text {critical }}$, and when the distance between two physical sensors is smaller or larger than $L_{\text {critical }}$, the coverage region of the virtual sensor is connected or disconnected. Given the source model, $L_{\text {critical }}$ decreases with the decision threshold. We place the two collaborating physical sensors $\left(s_{1}\right.$ and $\left.s_{2}\right)$ of a virtual sensor along the $\mathrm{X}$-axis of a Cartesian coordinate system (as shown in Fig. 4) with the mid point of the line segment $\overline{s_{1} s_{2}}$ at the origin of the system.

1) When $\mathcal{A}$ is connected: When the distance between two collaborating sensors is less than $L_{\text {critical }}, \mathcal{A}$ is connected, as shown in Fig. 4(a). We know that all the points on the boundary of $\mathcal{A}$ have the same sensing intensity, which we denote as

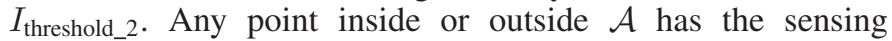

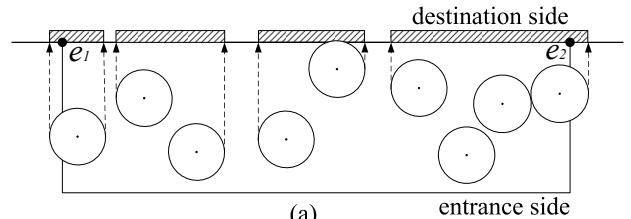

(a)

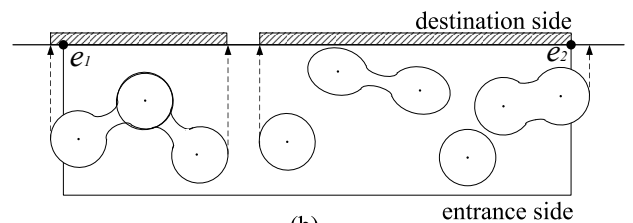

(b)

entrance side

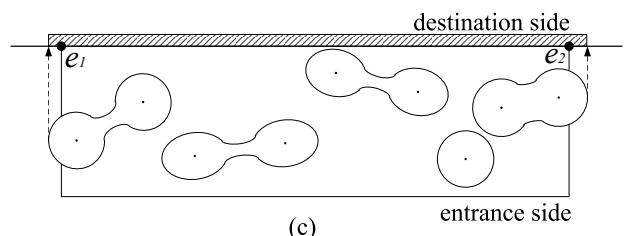

(c)

Fig. 2. Illustration of barrier information coverage. The destination side of the barrier is the line segment $\overline{e_{1} e_{2}}$. (a) Without sensor collaborations, the barrier is not covered. (b) Without well-planned sensor collaborations, the barrier is not covered. (c) Barrier is information-covered with well-planned sensor collaborations.
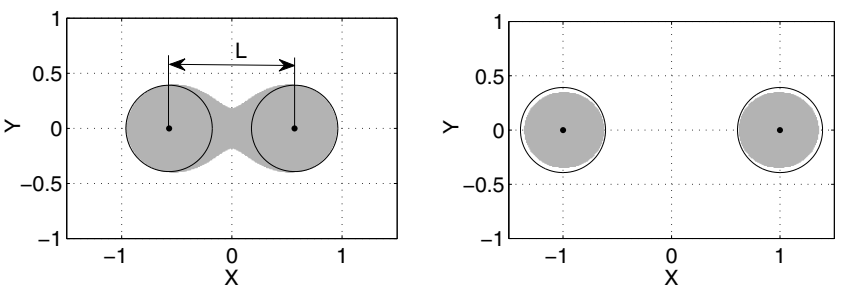

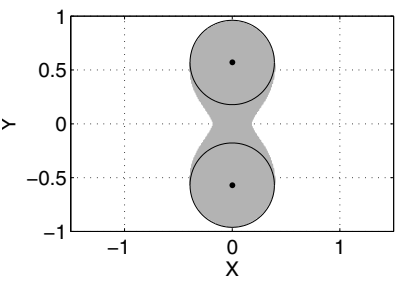

(c)

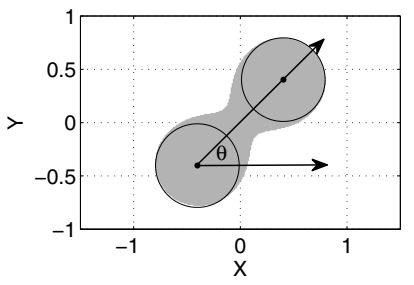

(d)
Fig. 3. Illustration of coverage regions of virtual sensors with their associated physical sensors at different positions. $\mathrm{X}$-axis is in parallel to the destination side of the barrier. The relative positions of two sensors are as follows: (a) $L=1.14$ units, $\theta=0$; (b) $L=2$ units, $\theta=0$; (c) $L=1.14$ units, $\theta=\frac{\pi}{2}$; and (d) $L=1.14$ units, $\theta=\frac{\pi}{4}$. We assume the noise follows a normal distribution with zero mean and standard deviation of $\frac{\sqrt{2}}{2} \mathrm{~mW}$. The decision threshold for a noColl sensor and a virtual sensor is $1.65 \mathrm{~mW}$ and $2.06 \mathrm{~mW}$, respectively. This is to make sure that $P_{f}=0.01$ for both noColl and virtual sensors. The radius of a noColl sensor's coverage region is 0.39 units.

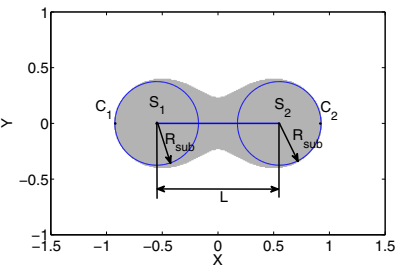

(a) when $L \leqslant L_{\text {critical }}$

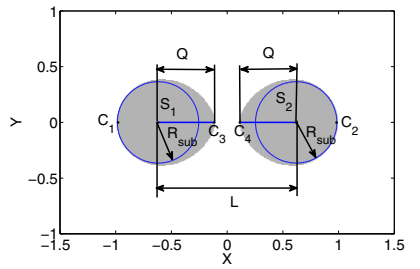

(b) when $L>L_{\text {critical }}$
Fig. 4. Illustration of $\mathcal{A}$ and $\mathcal{A}_{\text {sub }}$ of a virtual sensor. $\mathcal{A}$ is shown as the shaded region and $\mathcal{A}_{\text {sub }}$ is constituted of discs and line segments.

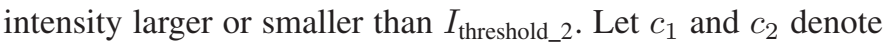
the leftmost and rightmost point of $\mathcal{A}$, respectively. It is easy to show (see [25] for proof details) that both $c_{1}$ and $c_{2}$ are along the $\mathrm{X}$-axis and $d\left(c_{1}, s_{1}\right)=d\left(c_{2}, s_{2}\right) \triangleq R_{\text {sub }}$. The sensing intensity at $c_{1}$ and $c_{2}$ is

$$
I_{\text {threshold_2 }}=I\left(d\left(c_{1}, s_{1}\right), d\left(c_{1}, s_{2}\right)\right)=I\left(d\left(c_{2}, s_{1}\right), d\left(c_{2}, s_{2}\right)\right) .
$$

Let $\odot_{s_{1}}$ and $\odot_{s_{2}}$ denote the disc centered at sensor $s_{1}$ and $s_{2}$, respectively, with a radius of $R_{\text {sub }}$. We have the following lemmas.

LEMMA 1: Both $\odot_{s_{1}}$ and $\odot_{s_{2}}$ are subsets of $\mathcal{A}$.

Proof: Consider any point $b$ inside or on the boundary of disc $\odot_{s_{1}}$. We have $d\left(b, s_{1}\right) \leqslant R_{\text {sub }}=d\left(c_{1}, s_{1}\right)$ and $d\left(b, s_{2}\right) \leqslant$ $d\left(b, s_{1}\right)+d\left(s_{1}, s_{2}\right) \leqslant d\left(c_{1}, s_{1}\right)+d\left(s_{1}, s_{2}\right)=d\left(c_{1}, s_{2}\right)$. From the definition of sensing intensity in Section III-B, we know

$$
I\left(d\left(b, s_{1}\right), d\left(b, s_{2}\right)\right) \geqslant I\left(d\left(c_{1}, s_{1}\right), d\left(c_{1}, s_{2}\right)\right)=I_{\text {threshold_2 }} .
$$

This means that $b$ is covered by the virtual sensor. As a result, the entire disc of $\odot_{s_{1}}$ is covered by the virtual sensor. Similarly, we can prove that the entire disc of $\odot_{s_{2}}$ also is covered by the virtual sensor.

Lemma 2: When $\mathcal{A}$ is connected, the line segment $\overline{s_{1} s_{2}}$ is a subset of $\mathcal{A}$. 
Proof: We prove this lemma by contradiction as follows. Assume there exists a point $b$ on the line segment $\overline{s_{1} s_{2}}$ that is not covered by the virtual sensor, i.e.,

$$
I\left(d\left(b, s_{1}\right), d\left(b, s_{2}\right)\right)<I_{\text {threshold_2 }} .
$$

Consider any other point $t$ on the line that is perpendicular to and intersects with $\overline{s_{1} s_{2}}$ at point $b$. Since $d\left(t, s_{1}\right) \geqslant d\left(b, s_{1}\right)$ and $d\left(t, s_{2}\right) \geqslant d\left(b, s_{2}\right)$, the sensing intensity at $t$ is

$$
I\left(d\left(t, s_{1}\right), d\left(t, s_{2}\right)\right) \leqslant I\left(d\left(b, s_{1}\right), d\left(b, s_{2}\right)\right)<I_{\text {threshold_2 }} .
$$

This means that $t$ also is not covered by the virtual sensor. As a result, none of the points on the line that is perpendicular to and intersects with $\overline{s_{1} s_{2}}$ is covered. Thus, $\mathcal{A}$ is disconnected, which contradicts with the assumption that $\mathcal{A}$ is connected.

Theorem 1: When $\mathcal{A}$ is connected, $\mathcal{A}_{\text {sub }}=\overline{s_{1} s_{2}} \cup \odot_{s_{1}} \cup \odot_{s_{2}}$ is a subset of $\mathcal{A}$.

The proof of this theorem is straightforward by applying Lemmas 1 and 2.

2) When $\mathcal{A}$ is disconnected: When the distance between two collaborating sensors is larger than $L_{\text {critical }}, \mathcal{A}$ becomes disconnected, as shown in Fig. 4(b). Note that Lemma 1 still holds in this situation. Let $c_{3}$ and $c_{4}$ denote the rightmost point in the left component and the leftmost point in the right component of $\mathcal{A}$, respectively. Again, it is easy to show (see [25] for details) that both $c_{3}$ and $c_{4}$ are along the $\mathrm{X}$-axis and $d\left(c_{3}, s_{1}\right)=d\left(c_{4}, s_{2}\right) \triangleq Q$. The sensing intensity at $c_{3}$ and $c_{4}$ is equal to $I_{\text {threshold_2 }}$ since both $c_{3}$ and $c_{4}$ are on the boundary of $\mathcal{A}$. We have the following lemma.

Lemma 3: When $\mathcal{A}$ is disconnected, line segments $\overline{s_{1} c_{3}}$ and $\overline{c_{4} s_{2}}$ are subsets of $\mathcal{A}$.

Proof: Consider any point $b$ on the line segment $\overline{s_{1} c_{3}}$ other than $s_{1}$ and $c_{3}$. We have $d\left(b, s_{1}\right)<d\left(c_{3}, s_{1}\right)<$ $d\left(c_{3}, s_{2}\right)<d\left(b, s_{2}\right)$. From the definition of sensing intensity in Section III-B, we know that the sensing intensity at $b$ is

$$
I\left(d\left(b, s_{1}\right), d\left(b, s_{2}\right)\right)=\left(\frac{1}{d\left(b, s_{1}\right)^{\alpha}}+\frac{1}{d\left(b, s_{2}\right)^{\alpha}}\right) \cdot \Phi,
$$

where $\Phi=\Omega d_{0}^{\alpha}$ is a constant. Because when $\alpha>0$ and $z>0$, the following always holds:

$$
\left.\left(\frac{1}{(x-z)^{\alpha}}-\frac{1}{x^{\alpha}}\right)^{\prime}\right|_{x>z}<0,
$$

we have:

$$
\begin{aligned}
& \frac{1}{\left(d\left(c_{3}, s_{1}\right)-d\left(c_{3}, b\right)\right)^{\alpha}}-\frac{1}{d\left(c_{3}, s_{1}\right)^{\alpha}} \\
&>\frac{1}{d\left(c_{3}, s_{2}\right)^{\alpha}}-\frac{1}{\left(d\left(c_{3}, s_{2}\right)+d\left(c_{3}, b\right)\right)^{\alpha}} \\
& \Longrightarrow \frac{1}{d\left(b, s_{1}\right)^{\alpha}}-\frac{1}{d\left(c_{3}, s_{1}\right)^{\alpha}}>\frac{1}{d\left(c_{3}, s_{2}\right)^{\alpha}}-\frac{1}{d\left(b, s_{2}\right)^{\alpha}} \\
& \Longrightarrow \frac{1}{d\left(b, s_{1}\right)^{\alpha}}+\frac{1}{d\left(b, s_{2}\right)^{\alpha}}>\frac{1}{d\left(c_{3}, s_{1}\right)^{\alpha}}+\frac{1}{d\left(c_{3}, s_{2}\right)^{\alpha}} \\
& \Longrightarrow I\left(d\left(b, s_{1}\right), d\left(b, s_{2}\right)\right)>I_{\text {threshold_2. }}
\end{aligned}
$$

We also know that points $s_{1}$ and $c_{3}$ are covered by the virtual sensor. This means that the entire line segment of $\overline{s_{1} c_{3}}$ is covered by the virtual sensor. Similarly, we can prove that the entire line segment of $\overline{c_{4} s_{2}}$ also is covered.

Theorem 2: When $\mathcal{A}$ is disconnected, $\mathcal{A}_{\text {sub }}=\overline{s_{1} c_{3}} \cup \overline{c_{4} s_{2}} \cup$ $\odot_{s_{1}} \cup \odot_{s_{2}}$ is a subset of $\mathcal{A}$.
The proof of this theorem is straightforward by applying Lemmas 1 and 3.

Note that the above theorems about $\mathcal{A}_{\text {sub }}$ always hold regardless of the values of $L$ and $\alpha$, while $R_{\text {sub }}$ and $Q$ vary with them. Since we are particularly interested in $\alpha=1$ or 2 in this paper, we give the corresponding results below:

- when $\alpha=1, R_{\text {sub }}=\frac{2 \Phi+\sqrt{4 \Phi^{2}+L^{2} I_{\text {threshold }}^{2}}}{2 I_{\text {threshold }}}-\frac{L}{2}$, and $Q=$ $\frac{L}{2}-\sqrt{\frac{L^{2}}{4}-\frac{L \Phi}{I_{\text {threshold }}}}$, and

- when $\alpha=2, R_{\text {sub }}=\frac{1}{2} \sqrt{\frac{4 \Phi+L^{2} I_{\text {threshold }}+4 \sqrt{\Phi I_{\text {threshold }} L^{2}+\Phi^{2}}}{I_{\text {threshold }}}}-$

$$
\frac{L}{2} \text {, and } Q=\frac{L}{2}-\frac{1}{2} \sqrt{\frac{4 \Phi+L^{2} I_{\text {threshold }}-4 \sqrt{\Phi I_{\text {threshold }} L^{2}+\Phi^{2}}}{I_{\text {threshold }}}},
$$

where $\Phi=\Omega d_{0}^{\alpha}$ is a constant.

\section{Determining $\left|\mathcal{P}_{\mathcal{A}_{\text {sub }}}\right|$}

Lемма 4: The barrier projection of $\mathcal{A}$ 's subset is a subset of the barrier projection of $\mathcal{A}$.

The proof of this lemma is straightforward and omitted due to space limitation. Therefore, $\mathcal{P}_{\mathcal{A}_{\text {sub }}}$ is a subset of $\mathcal{P}_{\mathcal{A}}$ and $\left|\mathcal{P}_{\mathcal{A}_{\text {sub }}}\right|$ is a lower bound for $\left|\mathcal{P}_{\mathcal{A}}\right|$. Assume that the coordinates of $s_{1}$ and $s_{2}$ are $\left(x_{1}, y_{1}\right)$ and $\left(x_{2}, y_{2}\right)$, respectively. We have the following results.

1) When $\mathcal{A}$ is connected: When $\mathcal{A}$ is connected, $\mathcal{A}_{\text {sub }}$ also is connected, and hence $\mathcal{P}_{\mathcal{A}_{\text {sub }}}$ is a single line segment. The $\mathrm{X}$-coordinates of the left and right end points of $\mathcal{P}_{\mathcal{A}_{\text {sub }}}$ are:

$$
\left\{\begin{array}{l}
x_{\ell}=\frac{x_{1}+x_{2}}{2}-R_{\text {sub }}-\frac{L}{2}|\cos \theta|, \\
x_{r}=\frac{x_{1}+x_{2}}{2}+R_{\text {sub }}+\frac{L}{2}|\cos \theta|,
\end{array}\right.
$$

where

$$
\left\{\begin{array}{l}
L=\sqrt{\left(x_{1}-x_{2}\right)^{2}+\left(y_{1}-y_{2}\right)^{2}}, \\
\theta=\arctan \left(\frac{y_{1}-y_{2}}{x_{1}-x_{2}}\right),
\end{array}\right.
$$

and $R_{\text {sub }}$ varies with $\alpha$ and is given in the previous section. Therefore, we have

$$
\left|\mathcal{P}_{\mathcal{A}_{\text {sub }}}\right|=x_{r}-x_{\ell}=2 R_{\text {sub }}+L|\cos \theta| .
$$

2) When $\mathcal{A}$ is disconnected: When $\mathcal{A}$ is disconnected, $\mathcal{A}_{\text {sub }}$ has two disconnected symmetric components, denoted as $\mathcal{A}_{\text {sub1 }}$ (containing $s_{1}$ ) and $\mathcal{A}_{\text {sub } 2}$ (containing $s_{2}$ ). However, $\mathcal{P}_{\mathcal{A}_{\text {sub }}}$ may be connected or disconnected, depending on the value of $\theta$. This means that the barrier projections of $\mathcal{A}_{\text {sub } 1}$ and $\mathcal{A}_{\text {sub2 }}$ may or may not overlap.

When $-\frac{\pi}{2} \leqslant \theta \leqslant \frac{\pi}{2}$, the X-coordinates of the left and right end points of the barrier projections of $\mathcal{A}_{\text {sub } 1}$ and $\mathcal{A}_{\text {sub } 2}$ are:

$$
\left\{\begin{array}{l}
x_{\ell 1}=\frac{x_{1}+x_{2}}{2}-\frac{L}{2} \cos \theta-R_{\text {sub }}, \\
x_{r 1}=\frac{x_{1}+x_{2}}{2}-\frac{L}{2} \cos \theta+\max \left\{Q \cos \theta, R_{\text {sub }}\right\}, \\
x_{\ell 2}=\frac{x_{1}+x_{2}}{2}+\frac{L}{2} \cos \theta-\max \left\{Q \cos \theta, R_{\text {sub }}\right\}, \\
x_{r 2}=\frac{x_{1}+x_{2}}{2}+\frac{L}{2} \cos \theta+R_{\text {sub }},
\end{array}\right.
$$

where $R_{\text {sub }}$ and $Q$ vary with $\alpha$ and are given in the previous section. We can see that when $\cos \theta \leqslant \frac{2 R_{\text {sub }}}{L}$, the barrier projections of $\mathcal{A}_{\text {sub } 1}$ and $\mathcal{A}_{\text {sub2 }}$ overlap and $\mathcal{P}_{\mathcal{A}_{\text {sub }}}$ becomes a single line segment. Therefore, we have

$$
\left|\mathcal{P}_{\mathcal{A}_{\text {sub }}}\right|= \begin{cases}x_{r 2}-x_{\ell 1}=2 R_{\text {sub }}+L \cos \theta & \text { if } \cos \theta \leqslant \frac{2 R_{\text {sub }}}{L}, \\ x_{r 2}-x_{\ell 2}+x_{r 1}-x_{\ell 1} & \\ =2 R_{\text {sub }}+2 \max \left\{Q \cos \theta, R_{\text {sub }}\right\} & \text { else. }\end{cases}
$$

Similar calculations can be done for $\theta>\frac{\pi}{2}$ or $\theta<-\frac{\pi}{2}$. 


\section{E. Numerical Results}

We propose to use $\left|\mathcal{P}_{\mathcal{A}_{\text {sub }}}\right|$ to approximate $\left|\mathcal{P}_{\mathcal{A}}\right|$. Now we use numerical study to evaluate the accuracy of such approximation, i.e., to evaluate the tightness of $\left|\mathcal{P}_{\mathcal{A}_{\text {sub }}}\right|$ as a lower bound for $\left|\mathcal{P}_{\mathcal{A}}\right|$. In this section and the rest of this paper, we will only show the results for $\alpha=1$ since the results for $\alpha=2$ show similar trends.

We plot $\left|\mathcal{P}_{\mathcal{A}}\right|$ and $\left|\mathcal{P}_{\mathcal{A}_{\text {sub }}}\right|$ for different $L$ and $\theta$ in Fig. 5(a) and (b), respectively. We have a couple of observations. Firstly, results show that $\left|\mathcal{P}_{\mathcal{A}}\right|$ and $\left|\mathcal{P}_{\mathcal{A}_{\text {sub }}}\right|$ are close to each other for different $L$ and $\theta$, meaning that $\left|\mathcal{P}_{\mathcal{A}_{\text {sub }}}\right|$ is a tight lower bound for $\left|\mathcal{P}_{\mathcal{A}}\right|$ and can approximate it well. Secondly, the length of the barrier projection of the coverage region of a virtual sensor reaches the maximum when the distance between its two collaborating physical sensors is about $L_{\text {critical }}$ and when the two collaborating sensors are along a line that is in parallel to the destination side of the barrier (i.e., $\theta=0$ ).

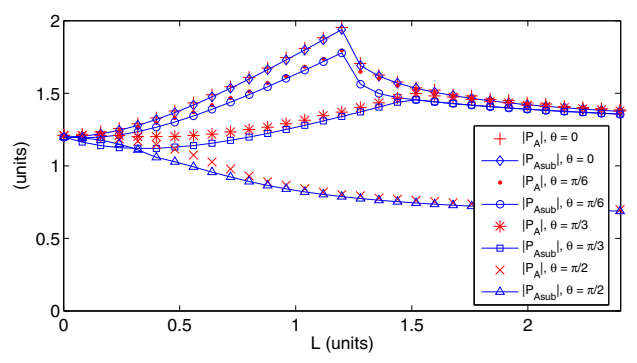

(a) $\left|\mathcal{P}_{\mathcal{A}}\right|,\left|\mathcal{P}_{\mathcal{A}_{\text {sub }}}\right|$ vs. $L\left(L_{\text {critical }}=1.19\right.$ units $)$

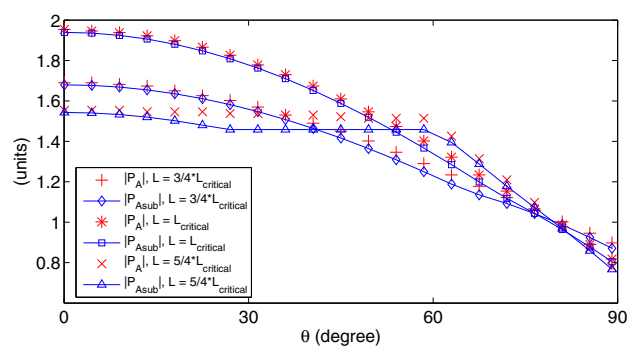

(b) $\left|\mathcal{P}_{\mathcal{A}}\right|,\left|\mathcal{P}_{\mathcal{A}_{\text {sub }}}\right|$ vs. $\theta\left(L_{\text {critical }}=1.19\right.$ units $)$

Fig. 5. The comparison between $\left|\mathcal{P}_{\mathcal{A}}\right|$ and $\left|\mathcal{P}_{\mathcal{A}_{\text {sub }}}\right|$ for different $L$ and $\theta$. Note that when $\theta=0,\left|\mathcal{P}_{\mathcal{A}}\right|=\left|\mathcal{P}_{\mathcal{A}_{\text {sub }}}\right|$.

Fig. 6 gives a more comprehensive view of the tradeoff between the length of the barrier projection of the coverage region and the sensor positions. In this figure, the first collaborating sensor sits at $(0,0)$ on the $\mathrm{X}-\mathrm{Y}$ plane at the bottom, where the $\mathrm{X}$-axis is parallel to the destination side of the barrier. The second sensor sits at $(x, y)$. Let $\left|\mathcal{P}_{\mathrm{N}}\right|$ denote the length of the barrier projection of the coverage region of these two sensors when they are not collaborating with each other. Fig. 6(a) plots the ratio of $\left|\mathcal{P}_{\mathcal{A}}\right|$ to $\left|\mathcal{P}_{\mathrm{N}}\right|$ when the second sensor sits at different positions. The contour curve at the bottom shows when the ratio is one. In other words, only when the second sensor sits inside the contour curve, the collaboration between these two sensors results in a longer length of the barrier projection of the coverage region, thus improving the barrier coverage performance. Fig. 6(b) plots the ratio of $\left|\mathcal{P}_{\mathcal{A}_{\text {sub }}}\right|$ to $\left|\mathcal{P}_{\mathrm{N}}\right|$ and we can see that the shape of the surface is similar to that in Fig. 6(a) and the contour curve approximates well the contour curve in Fig. 6(a).

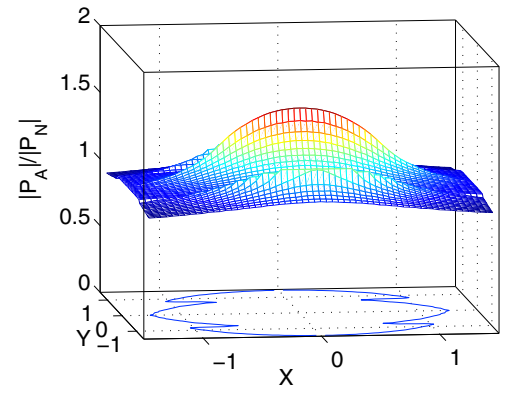

(a) $\frac{\left|\mathcal{P}_{\mathcal{A}}\right|}{\left|\mathcal{P}_{\mathrm{N}}\right|}$ vs. $(x, y)$ (contour curve shows when $\left.\left|\mathcal{P}_{\mathcal{A}}\right|=\left|\mathcal{P}_{\mathrm{N}}\right|\right)$

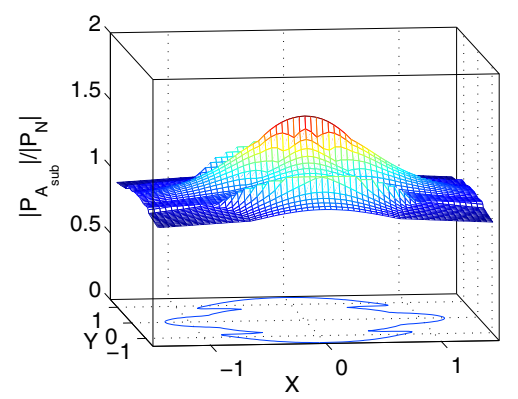

(b) $\frac{\left|\mathcal{P}_{\mathcal{A}_{\text {sub }}}\right|}{\left|\mathcal{P}_{\mathrm{N}}\right|}$ vs. $(x, y)$ (contour curve shows when $\left.\left|\mathcal{P}_{\mathcal{A}_{\text {sub }}}\right|=\left|\mathcal{P}_{\mathrm{N}}\right|\right)$

Fig. 6. Comparison of $\left|\mathcal{P}_{\mathcal{A}}\right|$ and $\left|\mathcal{P}_{\mathcal{A}_{\text {sub }}}\right|$ for different sensor positions. The first collaborating sensor sits at $(0,0)$ on the $\mathrm{X}-\mathrm{Y}$ plane and the second one sits at $(x, y) .\left|\mathcal{P}_{\mathrm{N}}\right|$ is the length of the barrier projection of the coverage region of these two sensors when they are not collaborating with each other.

\section{A Practical Solution to Find a Barrier INFORMATION COVERAGE SET (BICS)}

In this section, we describe a practical solution to find a set of active sensors to information-cover a barrier, i.e., a Barrier Information Coverage Set (BICS). The goal is to find a BICS consisting of as few active sensors as possible, thus prolonging the sensor network lifetime.

The problem of finding a BICS is more challenging than finding a conventional barrier coverage set under the $0 / 1$ disc sensing model. Under the $0 / 1$ disc sensing model, the coverage radius of an active sensor is usually fixed. By contrast, when considering information coverage under the probabilistic sensing model, the detection thresholds and consequently the sensors' coverage regions depend on the number of virtual and noColl sensors participating in the information coverage. In the case of virtual sensors, their coverage regions also depend on the relative positions of collaborating sensors. Moreover, the number of active sensors needed to constitute a BICS and the collaboration patterns for the virtual sensors are not predictable in practice. Thus, the underlying coverage graph is not known and it is very difficult, if not impossible, to enumerate all possible scenarios and find the optimal BICS that consists of minimum number of active sensors. For this reason, we propose a heuristic algorithm to find an appropriate BICS. Simulation results in Section VI show that the proposed algorithm can reduce the number of active sensors needed to cover the barrier and prolong the sensor network lifetime effectively.

\section{A. The Proposed Algorithm}

Our proposed algorithm to find a BICS requires the location information about all sensors and the barrier. The system's target false detection threshold and the target detection probability by a virtual or noColl sensor are also known. The pseudo-code of the algorithm is shown in Algorithm 1. 


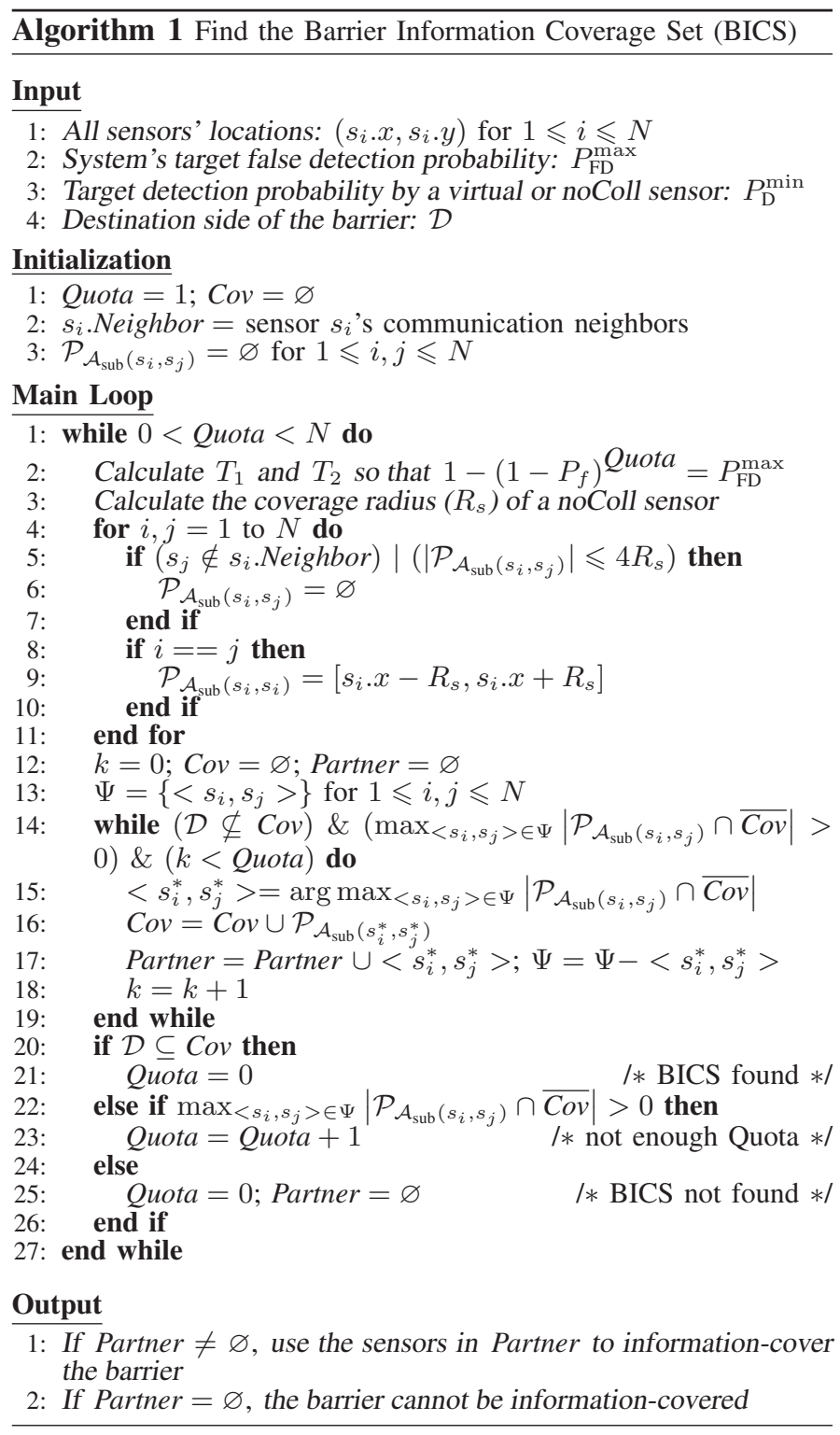

As shown in the pseudo-code, the algorithm operates by rounds and uses a variable called Quota, initially set to one and increased by one at each round, to denote the maximum number of virtual and noColl sensors to be considered at a round. Thus, at each round, based on the specified Quota, we can determine the detection thresholds for virtual and noColl sensors and consequently the coverage radius $\left(R_{s}\right)$ of a noColl sensor and the coverage region of a virtual sensor. Note that only the virtual sensors whose coverage region has a barrier projection longer than $4 R_{s}$ (i.e., twice that of a noColl sensor) may be part of a BICS. This is because a virtual sensor consists of two physical sensors and the collaboration between them consumes extra energy.

We use Partner to denote the set of selected virtual and noColl sensors and use Cov to denote the portion of the destination side of the barrier that has been covered by the barrier projections of the coverage regions of the selected sensors. At the beginning of each round, both Partner and Cov are set to empty. Then, under the Quota specified for the round, we search for a BICS in a greedy manner as follows. We always select a virtual or noColl sensor whose coverage region may increase $\mathrm{Cov}$ the most, and add it to Partner. A round ends when one of the following three events occurs:

- If Cov covers the entire destination side of the barrier, the algorithm halts and the Partner is output as a BICS.

- If Cov does not cover the destination side of the barrier, and if the number of selected virtual or noColl sensors is equal to the Quota specified for the round, while one of the remaining sensors may improve Cov further, we know that the current Quota is not large enough. Thus, Quota is increased by one and the algorithm continues.

- If Cov does not cover the destination side of the barrier, while none of the remaining sensors can increase Cov further, the algorithm halts and we claim that a BICS cannot be found.

Note that this algorithm may also be used to find the conventional barrier coverage set for non-collaborative sensors with little modification that only noColl sensors are considered when updating Partner.

\section{B. Energy Consumption for Sensor Collaborations}

After finding a BICS using the proposed algorithm, the sensor network uses the sensors selected in the BICS to information-cover the barrier. These sensors operate continuously until batteries die out. Then, a new BICS will be selected using the proposed algorithm and the sensors selected in the new BICS will be used to information-cover the barrier. The process continues until the barrier cannot be informationcovered by the remaining sensors in the network.

To operate as a virtual sensor, two physical sensors collaborate with each other by exchanging the sensed readings. If the sum of the sensed readings is larger than the detection threshold $\left(T_{2}\right)$, a detection decision is made. This also means that virtual sensors may improve the barrier coverage performance but at the expense of extra energy consumption for exchanging information between collaborating sensors. We propose a scheme to suppress the message exchanges between collaborating sensors as follows. If a physical sensor is to collaborate with another physical sensor to operate as a virtual sensor, it sends its sensed reading $(\mathbf{x})$ to its collaborator sensor only when $x \geqslant \frac{T_{2}}{2}$. This makes sense because if a virtual sensor makes a detection decision based on the sum of the sensed readings of two collaborating sensors, at least one of the readings should be larger than or equal to $\frac{T_{2}}{2}$. This way, the energy consumed for exchanging sensed readings between collaborating sensors may be reduced significantly. Next, we use an example to support the above explanation.

Consider a BICS consisting 12 virtual sensors and 17 noColl sensors to information-cover a barrier with the size of $20 \times 2$ units, as shown in Fig. 7(c). Assume that virtual and noColl sensors have the same false detection probability $\left(P_{f}\right)$. If the system's target false detection probability is $P_{\mathrm{FD}}^{\max }=0.05$ and the background noise follows a normal distribution with zero mean and standard deviation of $\frac{\sqrt{2}}{2} \mathrm{~mW}$, we can determine that the maximum false detection probability for each virtual or noColl sensor is $P_{f}^{\max }=0.0018$. Hence, according to Eq. (4), the decision threshold for each virtual sensor is $T_{2}=$ $2.915 \mathrm{~mW}$. Using the above message suppression scheme, a physical sensor only needs to send out its sensed reading when it exceeds $\frac{T_{2}}{2}=1.4575 \mathrm{~mW}$. This means that when there is no target in the barrier, a physical sensor will send its reading 
with a probability of $\frac{1}{2} \cdot \operatorname{erfc}\left(\frac{T_{2}}{2}\right) \approx 0.02$ at each sensing cycle, where $\operatorname{erfc}(\cdot)$ is the complementary Gauss error function. Suppose the sensing frequency is $25 \mathrm{~Hz}$. According to [26], the data rate of a MICA2 mote is $38.4 \mathrm{kbps}$, a packet with maximum payload has the size of 34 bytes, and maximum transmit power is $81 \mathrm{~mW}$. Then, energy consumption for sending a message is at most $\frac{34 \times 8 \times 81}{38400}=0.574 \mathrm{~mJ}$ and average power consumption for sending messages for collaborations is less than $\frac{34 \times 8 \times 81 \times 0.02 \times 25}{38400}=0.287 \mathrm{~mW}$. Such a small power consumption is almost negligible comparing to the power consumption (about $54 \mathrm{~mW}$ ) by other components of a MICA2 mote, including processor, radio receiver and sensor board.

\section{PERformance EVAluation}

We develop a custom simulator based on MATLAB and use it to evaluate the performance of our proposed algorithm. In the simulation, we randomly deploy $N$ sensors in a rectangular barrier with a length of 20 units and a width of 2 units, as shown in Fig. 7(a). Note that our algorithm works for any sensor distribution as long as the sensor locations after deployment are known. The system's target false detection probability is $P_{\mathrm{FD}}^{\max }=0.05$ and the target probability of detection by a virtual or noColl sensor is $P_{\mathrm{D}}^{\min }=0.9$. The signal amplitude of the target is $\Omega=100 \mathrm{~mW}$ and the small constant $d_{0}$ in the source model is set to 0.01 units. Assume that the background noise follows a normal distribution with zero mean and a standard deviation of $\frac{\sqrt{2}}{2} \mathrm{~mW}$. We only show the simulation results for the decay exponent of $\alpha=1$ because the results for $\alpha=2$ show similar trends.

\section{A. Coverage Snapshots}

Before discussing the simulation results in detail, we first present in Fig. 7 the coverage snapshots when our proposed algorithm is used to find BICS for a particular sensor network deployment. Fig. 7(a) shows 200 sensors deployed in a rectangular barrier with the size of $20 \times 2$ units. Fig. 7(b) shows that, without using virtual sensors, 54 noColl sensors are needed to cover the barrier. In contrast, with the help of sensor collaborations and virtual sensors, 12 virtual sensors (each of them consists of two collaborating physical sensors) and 17 noColl sensors are needed to cover the barrier. This means that only a total of 41 physical sensors need to be active to provide the desired barrier coverage. This example scenario clearly demonstrates (i) the effectiveness of information-coverage and sensor collaborations in reducing the number of active sensors needed to cover the barrier and (ii) the effectiveness of our proposed algorithm in finding a proper BICS. Similar observations are made throughout our simulation study.

Next, we present the simulation results for different scenarios, which are averaged over 200 different sensor network deployments unless specified otherwise. We compare two different coverage schemes: (i) Non-Collaborative - barrier coverage with noColl sensors only; and (ii) Collaborative barrier coverage with both virtual and noColl sensors.

\section{B. Comparison of $P_{B I C S}$}

Due to randomness in sensor deployment, it is impossible to guarantee that the barrier is covered with probability one with finite number of sensors, unless each active sensor can cover the entire barrier. However, physical limitations prohibit such large coverage region. This motivates us to study the barrier information coverage from a probabilistic perspective. Let $P_{\text {BICS }}$ denote the probability that a BICS can be found using our proposed algorithm. Fig. 8(a) plots $P_{\text {BICS }}$ for different number of sensors deployed in the barrier.

As shown in Fig. 8(a), $P_{\text {BICS }}$ increases monotonously with the number of deployed sensors for both schemes. In general, with fewer sensors deployed in the barrier, it is more difficult to find a BICS. Introduction of virtual sensors helps improve the barrier coverage performance. For example, when there are 140 sensors in the barrier, $P_{\mathrm{BICS}}$ for non-collaborative and collaborative schemes is about $12 \%$ and $57 \%$, respectively.

In Fig. 8(b), the number of deployed sensors to fixed to 140 and we vary the width of the barrier from 2 to 90 units and correspondingly the sensor network density from 3.5 to 0.078 per square unit. We can see that for the non-collaborative scheme, $P_{\mathrm{BICS}}$ is almost invariant with the barrier width. This is because a noColl sensor does not collaborate with others. As a result, the barrier coverage performance of the noncollaborative scheme does not depend on the area density of the noColl sensors in the two-dimensional barrier, but the line density of the noColl sensors projected on the destination side of the barrier, which does not change with the barrier width. In comparison, for the collaborative scheme, $P_{\mathrm{BICS}}$ decreases with the barrier width. This is because as the sensor network gets more sparse, it becomes more difficult for a sensor to find a proper collaborating partner to increase the coverage region. In the extreme cases when the barrier width is larger than about 64 units, sensors are too far away from each other to form any beneficial collaborations between them. Hence, no virtual sensors will be part of the BICS and the collaborative scheme becomes equivalent to the non-collaborative scheme.

\section{Comparison of Network Lifetime}

From Fig. 8(a), we know that in order to guarantee barrier coverage with a very high probability, a sufficiently large number of active sensors are needed. For the barrier simulated in our study, at least 250 active sensors are needed. Next, we vary the number of deployed sensors from 250 to 1000 and plot the number of disjoint BICS's for both schemes in Fig. 9(a). Clearly, comparing with the non-collaborative scheme, the
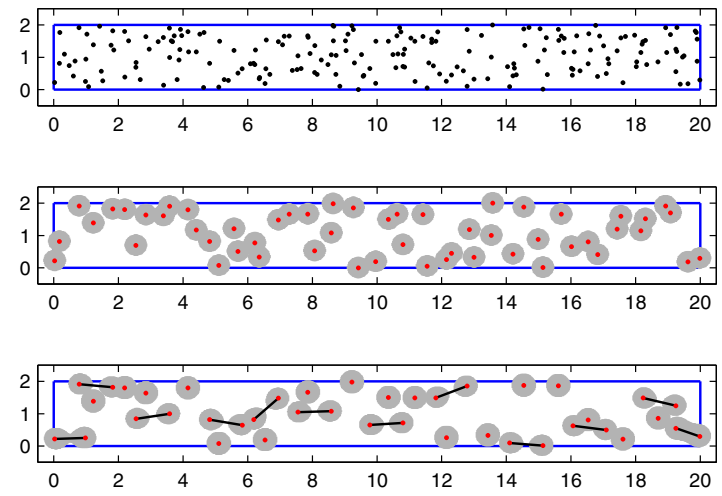

(c) A BICS consisting of both virtual and noColl sensors.

Fig. 7. Coverage snapshots for a given sensor network deployment. Shaded areas represent the coverage regions. Collaborating physical sensors associated to the same virtual sensor are connected with a solid line. (a) 200 sensors (shown as dots) are randomly deployed in a barrier with the size of $20 \times 2$ units. (b) Without using virtual sensors, the BICS selected by our algorithm consists of 54 noColl sensors. (c) By using virtual sensors, the BICS consists of 12 virtual sensors and 17 noColl sensors. 


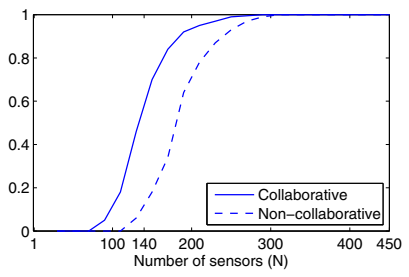

(a)

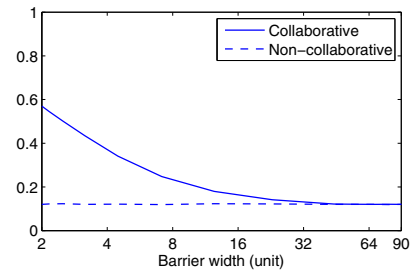

(b)
Fig. 8. Comparison of $P_{\text {BICS }}$ (a) $P_{\text {BICS }}$ vs. \# of deployed sensors $(N)$; and

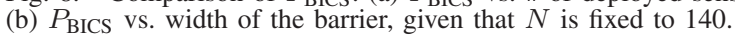

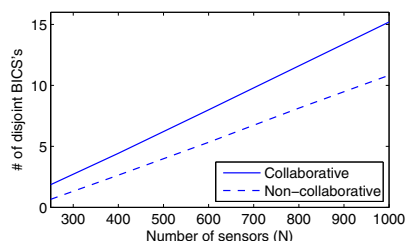

(a)

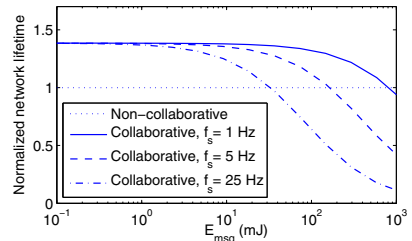

(b)
Fig. 9. Comparison of sensor network lifetime. (a) \# of disjoint BICS's vs. \# of deployed sensors $(N)$; and (b) Normalized network lifetime vs. energy consumption for sending one message.

collaborative scheme uses fewer physical sensors to cover the barrier with the help of virtual sensors and increases the number of disjoint BICS's by about $50 \%$ on average.

The number of disjoint BICS's is in direct relation to the sensor network lifetime. We define the network lifetime as the time when the remaining sensors in the network cannot cover the barrier any more. We assume that the lifetime of a BICS equals the lifetime of a virtual sensor because the physical sensors associated with a virtual sensor consumes more power than noColl sensors due to collaboration. Since each BICS can cover the barrier, the network lifetime is the sum of the lifetimes of successive BICS's selected by our algorithm. In this study, we only consider the energy consumption for sensing, collaborative decision making and sensor's regular operations e.g. CPU and radio. The energy consumed for a sensor to report its detection to the sink is not considered as it varies with the routing and aggregation methods used. Once the methods are known, they can be incorporated into our energy consumption study without much difficulty.

We assume that the power consumption for sensing, processing and receiving is $54 \mathrm{~mW}$ [26]. To illustrate the overhead of extra energy consumption for sensor collaboration, we assume that the target rarely appears in the barrier. Fig. 9(b) compares the network lifetime for different energy consumption for sending a message $\left(E_{\mathrm{msg}}\right)$ from 0.1 to $1000 \mathrm{~mJ}$ and for different sensing frequencies $\left(f_{s}\right)$ from 1 to $25 \mathrm{~Hz}$. Each point in the figure is normalized to the network lifetime with the noncollaborative scheme. The collaboration cost for using virtual sensors increases as $E_{\mathrm{msg}}$ and/or $f_{s}$ increase. As shown in the figure, when $E_{\mathrm{msg}}$ is low, the collaborative scheme always outperforms the non-collaborative scheme regardless of $f_{s}$. On the other hand, the network lifetime with the collaborative scheme drops below the non-collaborative scheme as $E_{\mathrm{msg}}$ increases and the drop occurs at a smaller $E_{\mathrm{msg}}$ value when $f_{s}$ is higher. This implies that the collaborative scheme works the best for sensor networks with small energy consumption for sending a message and low sensing frequency. Recall that in the example given in Section V-B, $E_{\mathrm{msg}}$ is at most $0.574 \mathrm{~mJ}$ for a MICA2 mote to send a message. Fig. 9(b) reads that the collaborative scheme can improve the lifetime of a MICA2- based sensor network deployed for barrier coverage by about $40 \%$ even at a relatively high sensing frequency of $25 \mathrm{~Hz}$, which is consistent with our discussion in the example.

\section{CONClusions And Future Work}

In this paper, we conduct extensive analytical and simulation studies on exploring sensors' collaborations and information fusion to improve the barrier coverage and extend the sensor network lifetime. We study the coverage region of a virtual sensor and its effect on the coverage performance. We derive an effective lower bound to approximate the projection length of the coverage region on the destination side of the barrier, based on which we propose a practical algorithm to operate the sensor network to information-cover the barrier with fewer active sensors, thus prolonging the network lifetime. The effectiveness of our solution is supported by numerical and simulation results. Future work includes design of a distributed algorithm for finding the barrier information coverage set and extension of the study to strong barrier coverage.

\section{REFERENCES}

[1] "Article id: 57702816." http://www.informationweek.com/news.

[2] B. Liu and D. Towsley, "A Study of the Coverage of Large-scale Sensor Networks," in Proc. IEEE MASS, Oct. 2004.

[3] S. Kumar, T. H. Lai, and A. Arora, "Barrier Coverage With Wireless Sensors," in Proc. ACM MobiCom, Aug. 2005.

[4] X. Bai, S. Kuma, D. Xua, Z. Yun, and T. H. Lai, "Deploying Wireless Sensors to Achieve Both Coverage and Connectivity," in Proc. ACM MobiHoc, May 2006.

[5] B. Liu, P. Brass, O. Dousse, P. Nain, and D. Towsley, "Mobility Improves Coverage of Sensor Networks," in Proc. ACM MobiHoc, May 2005.

[6] S. Kumar, T. Lai, and J. Balogh, "On k-coverage in a mostly sleeping sensor network," in Proc. ACM MobiCom, Philadelphia, PA, 2004.

[7] H. Zhang and J. Hou, "On deriving the upper bound of $\alpha$-lifetime for large sensor networks," in Proc. ACM MobiHoc, 2004.

[8] B. Liu, O. Dousse, J. Wang, and A. Saipulla, "Strong barrier coverage of wireless sensor networks," Proc. ACM MobiHoc, 2008.

[9] P. Balister, B. Bollobas, A. Sarkar, and S. Kumar, "Reliable density estimates for achieving coverage and connectivity in thin strips of finite length," ACM MobiCom, 2007.

[10] A. Chen, T.-H. Lai, and D. Xuan, "Measuring and guaranteeing quality of barrier-coverage in wireless sensor networks," Proc. ACM MobiHoc, 2008.

[11] S. Kumar, T. H. Lai, M. E. Posner, and P. Sinha, "Optimal sleep-wakeup algorithms for barriers of wireless sensors," Proc. IEEE BROADNETS, 2007.

[12] A. Chen, S. Kumar, and T. H. Lai, "Designing localized algorithms for barrier coverage," Proc. ACM MobiCom, 2007.

[13] X.-Y. Li, P.-J. Wan, and O. Frieder, "Coverage in wireless ad-hoc sensor networks," IEEE Transactions on Computers, vol. 52, no. 6, June 2003

[14] G. Veltri, Q. Huang, G. Qu, and M. Potkonjak, "Minimal and maximal exposure path algorithms for wireless embedded sensor networks," Proc. of ACM Sensys, 2003.

[15] S. Meguerdichian, F. Koushanfar, G. Qu, and M. Potkonjak, "Exposure In Wireless Ad-Hoc Sensor Networks," in Proc. ACM MobiCom, 2001

[16] J. Zhang, T. Yan, and S. Son, "Deployment Strategies for Differentiated Detection in Wireless Sensor Networks," in Proc. IEEE SECON, 2006.

[17] T.-L. Chin, P. Ramanathan, and K. K. Saluja, "Analytical Modeling of Detection Lantency in Mobile Sensor Networks," in Proc. IPSN, 2006.

[18] S. Aldosari and J. Moura, "Fusion in Sensor Networks with Communication Constraints," in Proc. IPSN, Apr. 2004.

[19] P. Varshney, Distributed Detection and Data Fusion. Springer, 1997.

[20] X. Luo, M. Dong, and Y. Huang, "On Distributed Fault-tolerant Detection in Wireless Sensor Networks," IEEE Transactions on Computers, vol. 55 , no. 1, pp. 58-70, 2006.

[21] T. Clouqueur, K. K. Saluja, and P. Ramanathan, "Fault Tolerance in Collaborative Sensor Networks for Target Detection," IEEE Transactions on Computers, vol. 53, no. 3, pp. 320-333, 2004.

[22] W. Wang, V. Srinivasan, K. C. Chua, and B. Wang, "Energy-efficient Coverage for Target Detection in Wireless Sensor Networks," in Proc. IPSN, Apr. 2007.

[23] G. Mazarakis and J. Avaritsiotis, "A prototype sensor node for footstep detection," In Proc. Euro. Workshop on Wireless Sensor Networks, 2005

[24] G. Succi, G. Prado, R. Gampert, T. Pedersen, and H. Dhaliwal, "Problem in seismic detection and tracking," In Proc. SPIE, vol. 4040, 2000.

[25] Tech. Rep., http://www.public.iastate.edu/Fgqyang.

[26] "Mica2 mote data sheet." http://www.xbow.com/ 